\title{
SYNTHESIS OF FLUORINE-18 LABELED 1,1-DIFLUORO-2,2-DICHLOROETHYL ARYL ETHERS BY 18F-FOR-19F EXCHANGE
}

\author{
Michael R. Kilbourn* and Raghu Subramanian \\ Division of Nuclear Medicine, Department of Internal Medicine, \\ University of Michigan, Ann Arbor, MI 48109
}

\begin{abstract}
SUMMARY
Fluorine-18 labeled 1,1-difluoro-2,2-dichloroethyl aryl ethers have been prepared by a facile ${ }^{18} \mathrm{~F}$-for- $19 \mathrm{~F}$ isotopic exchange reaction. The isotopic exchange proceeds in good to excellent yields (up to $85 \%$ ), and is dependent on reaction time, temperature, and concentration of reactants (aryl ether or fluoride ion). Substitution even occurs at room temperature in DMSO, and at elevated temperature $\left(100\right.$ to $\left.155^{\circ} \mathrm{C}\right)$ in DMSO or acetonitrile containing up to $20 \%$ water. Specific activities of $0.5-55 \mathrm{Ci} / \mathrm{mol}$ have been achieved, with the products obtained in pure radiochemical and chemical form.
\end{abstract}

KEY WORDS: $\quad\left[{ }^{18} \mathrm{~F}\right]$ fluoride ion, isotopic exchange,

nucleophilic substitution, perfluoroethers

\section{INTRODUCTION}

[18F]Fluoride ion has become a readily available, widely used precursor for radiopharmaceutical synthesis, and has been applied to numerous aliphatic and aromatic nucleophilic substitution reactions $(1,2)$. As part of our interests in labeling of organofluorine compounds with fluorine-18, we report here the simple, 
unprecedented synthesis of carrier-added fluorine-18 labeled 1,1-difluoro-2,2dichloroethyl aryl ethers by a remarkably facile ${ }^{18} \mathrm{~F}$-for- ${ }^{19} \mathrm{~F}$ exchange reaction.

\section{EXPERIMENTAL}

Materials and Methods. No-carrier-added [18F]fluoride ion was produced by proton irradiation of 95-97\% oxygen-18 enriched water (Isotec) held in an all-silver cyclotron target (3). Acetonitrile and dimethylsulfoxide were obtained in anhydrous form (Aldrich Chem. Co.) and used without further purification. The 1,1-difluoro2,2-dichloroethyl aryl ethers (1-4) were prepared by condensation of the appropriate sodium phenoxide with 1,1-difluoro-2,2-dichloroethylene under phase transfer conditions $(4,5)$. TLC was done using plastic-backed silica gel plates (Merck) and one of two solvent systems: System $A=$ hexane; System $B=7 / 3$ hexane/ethylacetate. HPLC was done using a Phenomenex C18 column, $0.45 \times 15 \mathrm{~cm}, 60: 40 \mathrm{CH}_{3} \mathrm{CN}: 0.065$ $\mathrm{M} \mathrm{NH}{ }_{4} \mathrm{OAc}, 1 \mathrm{ml} / \mathrm{min}$ : detection by $\mathrm{UV}(264 \mathrm{~nm}$ ) and radioactivity (Beckman Model 170 flow detector).

General Procedure for $\left[{ }^{18}\right.$ F]Fluorinations. Aqueous [18F]fluoride ion was combined with potassium carbonate $(1.8 \mathrm{mg}$ ) and Kryptofix-2,2,2 (10 $\mathrm{mg})$ and the water evaporated ( $100^{\circ} \mathrm{C}, \mathrm{N}_{2}$ flow). Acetonitrile was added and evaporated to remove traces of water. The residue was dissolved in $1 \mathrm{ml}$ of anhydrous solvent, and $100 \mathrm{ml}$ aliquots used in subsequent $\left[{ }^{18} \mathrm{~F}\right]$ fluorination reactions. The $\left[{ }^{18} \mathrm{~F}\right]$ fluorinations were performed by adding $100 \mu \mathrm{l}$ of a solution of ether (1-4) to the [18F]fluoride solution, then heating (various temperatures and reaction times: see Table 1). Reactions were quenched by addition of $2 \mathrm{ml}$ water, and the aqueous mixture extracted with $4 \mathrm{ml}$ of diethyl ether or 4/1 diethyl ether/ethylacetate. The organic layers were then analyzed by TLC and the yields of fluoride exchange (radioactivity in organic phase/total radioactivity) corrected for $\left[{ }^{18} \mathrm{~F}\right]$ fluoride ion (if any) extracted into the organic solution. Identity and purity (chemical and radiochemical) were also determined by injection of the isolated products on HPLC. In some instances, aliquots from reactions done in acetonitrile were directly spotted on TLC plates, or were diluted with $60: 40 \mathrm{CH}_{3} \mathrm{CN}: \mathrm{H}_{2} \mathrm{O}$ and directly injected on the HPLC. 
The $R_{f}$ values (TLC) and $R_{t}$ times (HPLC) for the four ethers were as follows:

(1): TLC, $A, R_{f}=0.28 ; B R_{f}=0.61: H P L C, R_{t}=13.9 \min$.

(2): TLC, $A, R_{f}=0.27 ; B R_{f}=0.43:$ HPLC, $R_{t}=14.0 \mathrm{~min}$.

(3): TLC, $A, R_{f}=0.05 ; B R_{f}=0.37: H P L C, R_{t}=12.8 \mathrm{~min}$.

(4): TLC $A, R_{f}=0.05 ; B R_{f}=0.43: H P L C, R_{t}=12.9$ min.

For some reactions (see Table 1 ), the $\left[{ }^{18} \mathrm{~F}\right]$ fluoride ion was prepared as the tetrabutylammonium fluoride salt (substitution of TBAF for $\mathrm{K}_{2} \mathrm{CO}_{3} / \mathrm{Kryptofix} \mathrm{2,2,2)}$ or as a polymer-bound species, using an aminopyridine-substituted polystyrene polymer as previously described (6).

\section{RESULTS AND DISCUSSION}

The reaction of $[18 \mathrm{~F}]$ fluoride ion (as the soluble $\mathrm{K}^{+} /$Kryptofix salt, tetrabutylammonium salt, or resin-bound) with 1,1-difluoro-2,2-dichloroethyl aryl ethers 1.4 proceeds with $18 \mathrm{~F}$-for- $19 \mathrm{~F}$ substitution to give moderate to excellent yields of the $\left[{ }^{18} \mathrm{~F}\right]$ ethers (Table 1 ). The isotopic exchange reaction is dependent on temperature, length of reaction, concentration of substrate (aryl ether), and substituent on the aryl ring. Highest yields, and greatest reactivity, was exhibited by the 3-nitrophenyl ether 3, with lowest yields obtained with the 4-fluorophenyl ether 2.

Dipolar aprotic solvents appear most useful for this exchange reaction. Addition of non-polar solvents, such as chloroform or mesitylene, reduce [18F]fluorination yields. Most surprisingly, however, were the nearly $50 \%$ yields obtained in solutions of DMSO or acetonitrile containing up to $20 \%(\mathrm{v} / \mathrm{v})$ of water. Exchange of $\left[{ }^{18}\right.$ F]fluoride with 3 also takes place in the absence of any solvent, with the yield $\left(44 \%, 30 \mathrm{~min}, 155^{\circ} \mathrm{C}\right.$ ) perhaps limited by the solubility (unknown) of the $\mathrm{K}[18 \mathrm{~F}] \mathrm{F} / \mathrm{Kryptofix}$ reagent in the neat ether solution. The exchange reaction is not dependent on the nature of the cation $\left(\mathrm{K}^{+}\right.$, tetrabutylammonium, or resin) as essentially identical results were obtained with all three sources of [18F]fluoride ion.

The remarkable ease of ${ }^{18} \mathrm{~F}$-for- ${ }^{19} \mathrm{~F}$ exchange in these 1,1-difluoro-2,2dichloroethyl aryl ethers was surprising. Although isotopic fluorine exchange is precedented with both alkyl and aryl fluorides, previous attempts to effect isotopic fluorine exchanges in perfluoroalkyl compounds have met limited success and have 
Table 1. Yields of 1,1-[18F]difluoro-2,2,-dichloroethyl aryl ethers obtained by isotopic fluorine exchange. Yields are decay-corrected.
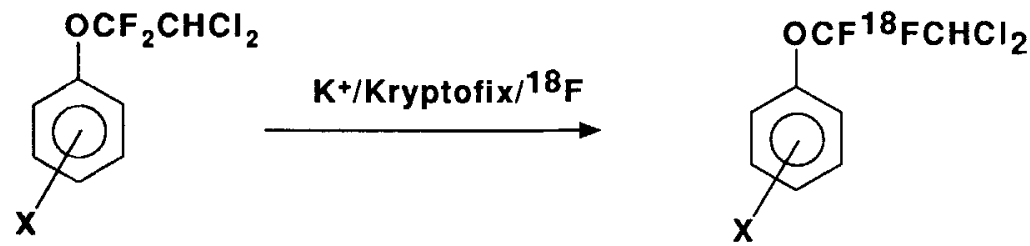

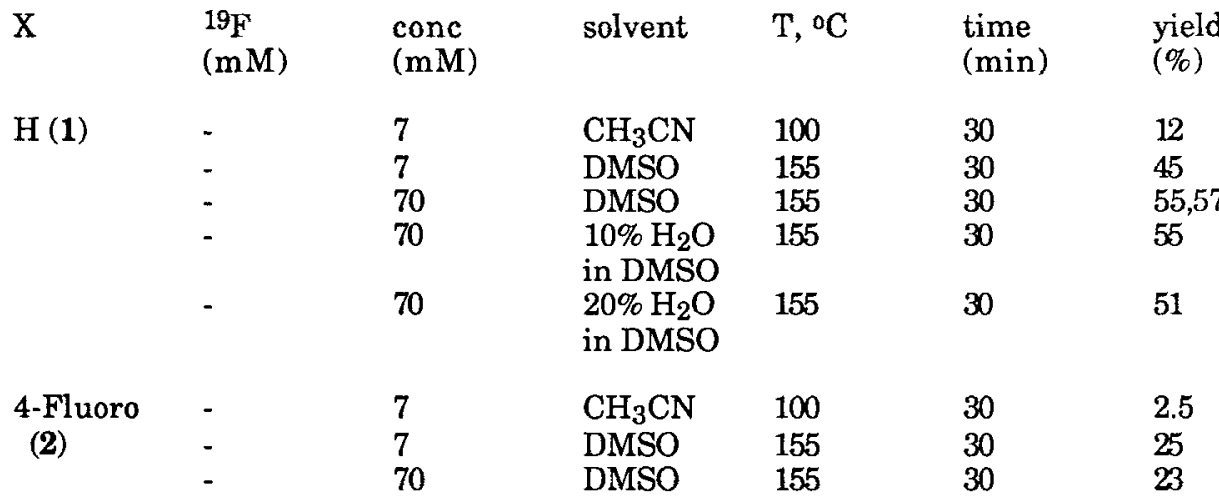

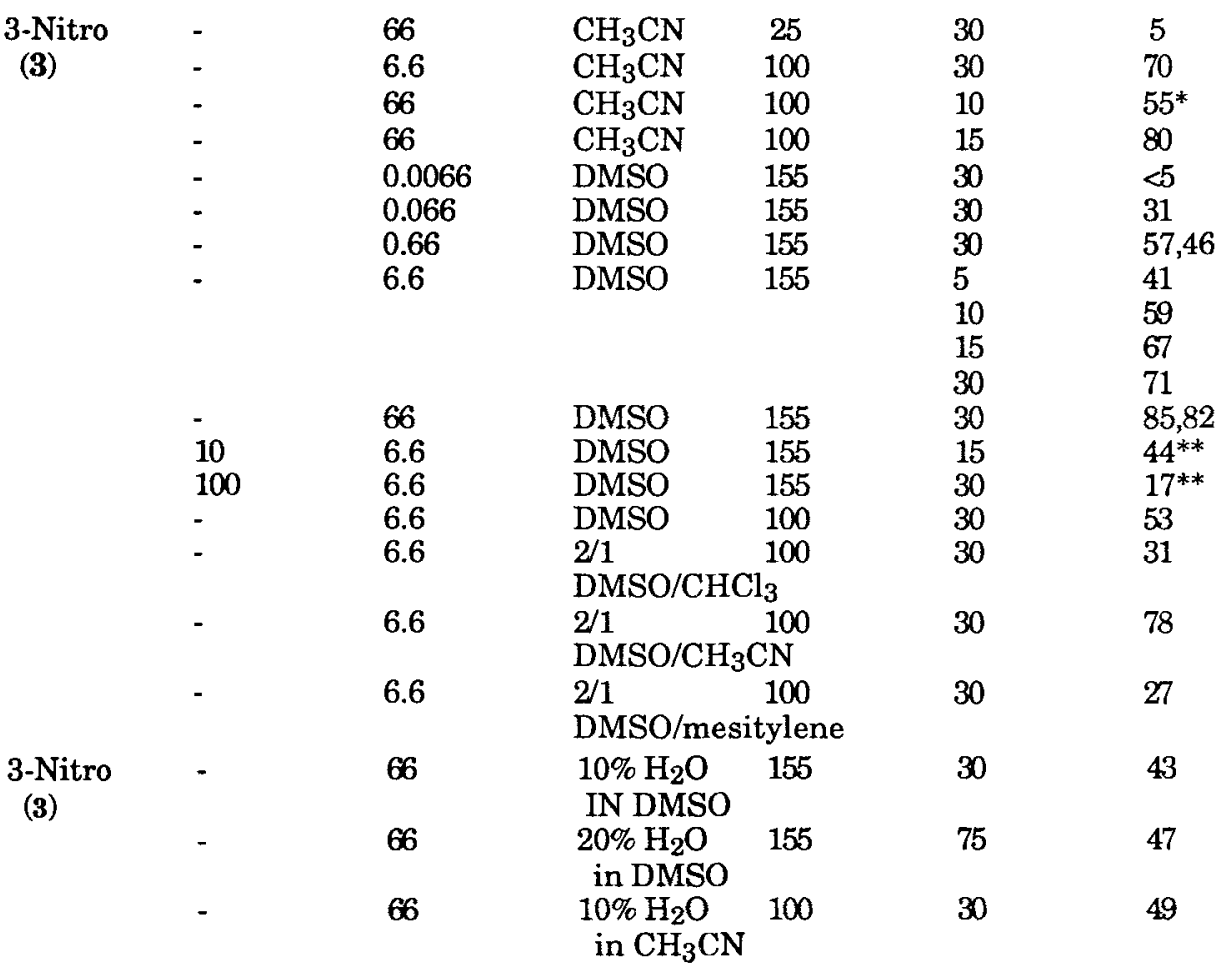


Table 1, cont'd.

\begin{tabular}{|c|c|c|c|c|c|c|}
\hline $\mathrm{X}$ & $\begin{array}{l}19 \mathrm{~F} \\
(\mathrm{mM})\end{array}$ & $\begin{array}{l}\text { conc } \\
(\mathrm{mM})\end{array}$ & solvent & $\mathrm{T},{ }^{\circ} \mathrm{C}$ & $\begin{array}{l}\text { time } \\
(\min )\end{array}$ & $\begin{array}{l}\text { yield } \\
(\%)\end{array}$ \\
\hline & - & 66 & $\begin{array}{l}20 \% \mathrm{H}_{2} \mathrm{O} \\
\text { in } \mathrm{CH}_{3} \mathrm{CN}\end{array}$ & 100 & 30 & 12 \\
\hline 4-Nitro & - & 6.6 & $\mathrm{CH}_{3} \mathrm{CN}$ & 100 & 30 & 31 \\
\hline (4) & - & 6.6 & DMSO & 155 & $\begin{array}{l}5 \\
10 \\
15 \\
30\end{array}$ & $\begin{array}{l}23 \\
27 \\
34 \\
30\end{array}$ \\
\hline
\end{tabular}

* [18F]fluoride prepared in polymer-supported form

**[18F]fluoride prepared as tetrabutylammonium salt

required high reaction temperatures $(7,8,9)$. The isotopic exchange reported here occurs even at room temperature, albeit in low yield. Assignment of the products as due to isotopic fluorine exchange reaction, rather than ring substitutions of nitro or fluoro groups, was based on the reactivity of the unsubstituted aryl ether 1 , as well as TLC and HPLC analysis showing the labeled product from reaction of 4-nitrophenyl ether 4 was identical to the unlabeled ether, and quite separate from the alternative product involving ring fluorination (compound 3). Perfluoroalkyl ethers have been previously examined for effects on ring reactivity, and have been classified as halogen-like (10), and thus would not be expected to be activating towards nucleophilic substitutions of phenyl rings. Fluorine-18 substitution for the fluorine, rather than one of the chlorines, follows from the reactivity of $\alpha$-fluoroalkyl ethers towards $\mathrm{AlCl}_{3}$ to produce $\alpha, \alpha, \alpha$-trichloroperfluoroethers (11), substitution of 1,1 difluoro-2,2-dichloroethyl aryl ethers by bromide ion $\left(\mathrm{AlBr}_{3}\right)$ to yield 1,1-dibromo-2,2dichloroethyl ethers (4), and nucleophilic attack by sodium ethylate on ethoxy-1,1difluoro-2,2,-dichloroethane to give the orthoester (substitution of ethoxide for fluoride, rather than chloride (12)). [18F]Fluoride ion substitution of a chlorine is precedented with alkyl chlorides (13), but the presence of the two fluorine substituents on the alpha position would be expected to significantly decrease the reactivity of the chlorines toward simple nucleophilic substitution. In all cases the radiolabeled products were identical chromatographically to the starting materials, and we could not identify any products which might have arisen by F-for-Cl substitution, nor any products which might arise by rearrangement reactions. 
Although the 1,1-difluoro-2,2-dichloroethyl ethers are subject to both rearrangement and hydrolysis under strongly basic reaction conditions $(4,5)$, we did not find chemical or radiochemical by-products which would support such reactions under the conditions utilized here for the isotopic exchange. Our preparations of [18F]fluoride ion in organic solvents (using $\mathrm{K}_{2} \mathrm{CO}_{3} / \mathrm{Kryptofix}$, tetrabutylammonium hydroxide, or anion exchange resin) are all quite suitable for use with substrates which are sensitive to basic conditions, as evidenced by good yields in the fluorination of the triflate precursor to 2-deoxy-2-[18F]fluoro-D-glucose.

The reactions described here yield, of course, the labeled aryl ethers in carrieradded form, with specific activities in the range of $0.5-55 \mathrm{Ci} / \mathrm{mol}$. Most experiments in Table 1 were done with small amounts $(100 \mu \mathrm{Ci}$ or less) of [18 F]fluoride ion, although we have prepared multimillicurie amounts of the compounds 1 and 3 in fluorine-18 form. The use of high initial levels of [ $\left.{ }^{18} \mathrm{~F}\right]$ fluoride ion, coupled with low amounts of aryl ether $(0.1 \mu \mathrm{mol})$ might lead to fluorine-18 labeled products of higher (50-100 Ci/mmol) specific activity. The products are obtained in very high chemical purity (i.e., no decomposition or side reactions) and can be easily separated from unreacted [18F]fluoride ion (silica gel column). 1,1-Difluoro-2,2-dichloroethyl ethers are interesting chemical structures. Recently investigated as phenol protecting groups, they are remarkably stable to a wide variety of reaction conditions and reagents (4) The possible uses of such aryl ethers to prepare radiopharmaceuticals (for those cases where high specific activity is not necessary) are under investigation.

Acknowledgements. This work was supported by Department of Energy Grant DE-AC02-76EV02031 and NIH Training Grant T32-CA09015 (to R.S.).

\section{REFERENCES}

1. Berridge M.S. and Tewson T.J. - Appl. Radiat. Isot. 37:685 (1986).

2. Fowler J.S. and Wolf A.P., The synthesis of carbon-11, fluorine-18 and nitrogen-13 labeled radiotracers for biomedical applications. Technical Information Service, U.S. Department of Energy,Monograph NAS-NS-3201, $124 \mathrm{pp}, 1982$

3. Mulholland G.K., Hichwa R.D., Kilbourn M.R., Moskwa J. - J. Labeled Compd. Radiopharm. 26: 140 (1989). 
4. Subramanian R - Ph.D. Thesis, State Univ. of New York-Stony Brook, 1988.

5. Subramanian R. and Johnson F. - J. Org. Chem. 50: 5430 (1985).

6. Mulholland G.K., Magner T.J., Jewett D.M., and Kilbourn M.R. - J. Labeled Compd. Radiopharm. 26: 378 (1989).

7. Boggs J.E., Van Artsdalen E.R., and Brosi A.R., - J. Amer. Chem. Soc. 77: 6505 (1955).

8. Gens T.A., Brost A.R., and Van Artsdalen E.R. - J. Amer. Chem. Soc. 79:1001 (1957).

9. Ido T., Irie T., and Kasida Y. - J. Labeled Compd. Radiopharm. 16: 153 (1979).

10. Sheppard W.A. - J. Amer. Chem. Soc. 85:1314 (1963).

11. Tiers G. - J. Amer. Chem. Soc. 77: 4837 (1955).

12. Tarrant P. and Brown H.C. - J. Amer. Chem. Soc. 73:1781 (1951)

13. Luxen A., Barrio J.R., Satyamurthy N., Bida G.T., and Phelps M.E. - J. Fluorine Chem. 36:83 (1987) 